\title{
Clinical significance of dual-time-point 18F-FDG PET imaging in resectable non-small cell lung cancer
}

\author{
Katsuhiko Shimizu $^{1} \cdot$ Riki Okita $^{1} \cdot$ Shinsuke Saisho $^{1} \cdot$ Takuro Yukawa $^{1}$. \\ Ai Maeda ${ }^{1} \cdot$ Yuji Nojima $^{1} \cdot$ Masao Nakata $^{1}$
}

Received: 6 April 2015/ Accepted: 30 July 2015/Published online: 8 August 2015

(c) The Author(s) 2015. This article is published with open access at Springerlink.com

\begin{abstract}
Objective The maximal standardized uptake value (SUVmax) of pulmonary lesions on dual-time-point (DTP) fluorodeoxyglucose positron emission tomography (FDGPET) has been shown to be useful for differentiation between malignant and non-malignant pulmonary lesions, and also to be of value for intrathoracic nodal staging of non-small cell lung cancer (NSCLC). However, a few NSCLC lesions have been found to show decreased FDG uptake on delayed images, and the significance of this finding remains unknown.

Patients and methods We conducted a retrospective review of the data of 284 patients with NSCLC who underwent DTP FDG-PET before surgery. Cases of adenocarcinoma in situ and minimally invasive adenocarcinoma were excluded, because these lesions show little FDG uptake. Each patient was scanned at 60 min (early acquisition; SUV-E) and $115 \mathrm{~min}$ (delayed acquisition; SUV-D) after the radiopharmaceutical injection. The intratumoral retention index (RI) of 18F-FDG was measured for each examination by the DTP method. Recurrence-free survival (RFS) was determined by the KaplanMeier method and compared in relation to the SUV-E, SUV-D, and RI by univariate and multivariate analysis using models including the clinico-pathological prognostic factors.

Results Of the 284 cases, the $\mathrm{RI} \leq 0$ was in 49 cases
\end{abstract} $(17.3 \%)$. This group of patients showed lower values of

Katsuhiko Shimizu

kshimizu@med.kawasaki-m.ac.jp

1 Department of General Thoracic Surgery, Kawasaki Medical School, 577 Matsushima, Kurashiki, Okayama 701-0192, Japan
SUV-E and SUV-D, a smaller tumor size, and a lower rate of lymphatic invasion or vascular invasion. It was particularly noteworthy that lymph node metastasis was not histopathologically confirmed in any of these patients. Univariate analysis identified the RI, SUV-E and SUV-D, besides age, tumor size, lymph node metastasis, and tumor differentiation grade as predictors of the RFS. On the other hand, multivariate analysis identified the RI and lymph node metastasis, but not the SUV-E and SUV-D, as independent predictors of the RFS.

Conclusions This study demonstrated that DTP FDGPET of the primary tumor in NSCLC can be useful to predict the RFS of the patients. In addition, this method may also be useful to predict the presence/absence of intrathoracic lymph node metastasis in these patients.

Keywords Non-small cell lung cancer - Lymph node metastasis · Dual-time-point imaging 18F-FDG PET . Retention index

\section{Introduction}

Fluorodeoxyglucose positron emission tomography (FDGPET) has become an important tool for the diagnosis and staging of non-small cell lung cancer (NSCLC) [1]. The maximal standardized uptake values (SUVmax) on FDGPET is calculated as the ratio of the activity in the tissue per unit volume to the injected dose by body weight, and is widely used because of the simplicity of its measurement [2]. In NSCLC patients, the SUVmax values of the primary tumors have been known to be correlated with the disease stage, nodal status, histological type, tumor differentiation grade, and rate of progression of the tumors [3-5]. In addition, high SUVmax values have been reported as being 
among the poor prognostic factors in patients with NSCLC [5-7].

Several studies have reported that determination of the SUVmax of pulmonary lesions on dual-time-point (DTP) FDG-PET is useful for differentiation between malignant and non-malignant pulmonary lesions [8-10]. Moreover, several investigators have shown that determination of the SUVmax of the lymph nodes on DTP FDG-PET improved the diagnostic ability of this imaging modality for intrathoracic nodal staging in NSCLC patients [11-13]. In general, most malignant lesions, including primary tumors and lymph nodes, also show increased FDG uptake on the delayed-phase images.

However, it has been reported that some NSCLC lesions actually show decreased FDG uptake on the delayed-phase images [14]. The significance of such decreased FDG uptake of NSCLC lesions remains unknown. In the present study, we analyzed the diagnostic impact of DTP FDGPET for intrathoracic nodal staging and also its prognostic impact in patients with resectable NSCLC.

\section{Patients and methods}

\section{Study population}

We conducted this retrospective study in a total of 284 patients, who had undergone DTP FDG-PET before surgery, which consisted of surgical resection with lymph node dissection or sampling, at the Kawasaki Medical School Hospital between 2007 and 2013. None of the patients had received either radiotherapy or chemotherapy prior to the surgery. The TNM stage was determined according to the revised criteria published in 2009. The histological diagnosis of the tumors was based on the criteria of the IASLC/ATS/ERS proposed in 2011 [15]. Cases of adenocarcinoma in situ and minimally invasive adenocarcinoma were excluded, because these lesions show little uptake of FDG [16]. This study was conducted with the approval of the institutional Ethics Committee of Kawasaki Medical School (No.1323). Follow-up information until either recurrence or December 2014 was obtained from the medical records.

\section{Fdg-pet}

All PET/CT examinations were performed with a dedicated PET/CT scanner (Discovery ST Elite; GE Healthcare, Japan). The axes of the multidetector CT and PET systems were mechanically aligned so that the patient could be moved from the CT to the PET scanner gantry by simply changing the position of the examination table. The resultant PET and CT scans were coregistered with hardware. PET/CT scanning was performed at 60 and $115 \mathrm{~min}$ after the intravenous injection of $150-220 \mathrm{MBq}$ of ${ }^{18}$ FDG (FDGscan, Universal Giken, Nihon Mediphysics, Tokyo, Japan). The regions of interest (ROI) were placed three-dimensionally over the lung cancer nodules. Semiquantitative analysis of the images was performed by measuring the SUVmax of the lesions. The SUVs were calculated using the following equation: tumor activity concentration/(injected dose/body weight). Therefore, the SUVmax measurements of the primary tumor were obtained both on early- (SUV-E) and delayed scans (SUVD). In this study, we analyzed the "no integration of FDG" as "zero". In addition, all the cases with SUV-E value of zero also had SUV-D value of zero. Furthermore, we calculated the retention index (RI) from the SUVmax values by the following formula: RI $(\%)=($ SUV-D-SUV-E) $/$ SUV-E $\times 100$ when SUV-E $>0$, and RI $(\%)=0$ when $\mathrm{SUV}-\mathrm{E}=0$. RI is really impossible calculation with SUV$E$ and SUV-D value of zero, therefore, we considered the value as zero.

\section{Statistical analysis}

Statistical analysis was performed for examining the significances of differences and the possible correlations between the RI on DTP FDG-PET, and the clinicopathological features; Fisher's exact test or the $\chi^{2}$ test was used, as appropriate. An unpaired $t$ test was used for comparison of continuous data. The prognostic evaluation was based on the recurrence-free survival (RFS). RFS was defined as the time from the date of surgery until lung cancer recurrence or non-lung cancer death. The survival curves were estimated using the Kaplan-Meier method, and differences among the curves were evaluated by the log-rank test. Univariate and multivariate analysis were performed using Cox proportional hazard models. All the statistical analyses were conducted using the SPSS software (Version 17.0; SPSS Incorporation, Chicago, IL). All statistical tests were two-sided, and probability values of $<0.05$ were regarded as denoting statistical significance.

\section{Results}

\section{Clinical characteristics}

The characteristics of the patients are summarized in Table 1. The patients ranged in age from 37 to 83 years (mean, 70.4), and there were 195 men and 89 women. The majority of patients $(184,64.8 \%$ ) had adenocarcinoma, while $71(25.0 \%)$ had squamous cell carcinoma, 16 (5.6\%) had large cell carcinoma, and 13 (4.6\%) had other histological types. Pathological N0 disease was confirmed 
Table 1 Patient characteristics $(n=284)$

\begin{tabular}{|c|c|c|}
\hline Variable & Number & $\%$ \\
\hline Age, mean \pm SD & $70.4 \pm 8.9$ & \\
\hline \multicolumn{3}{|l|}{ Sex } \\
\hline Male & 195 & 68.6 \\
\hline Female & 89 & 31.4 \\
\hline \multicolumn{3}{|l|}{ Histology } \\
\hline Adenocarcinoma & 184 & 64.8 \\
\hline Squamous cell & 71 & 25.0 \\
\hline Large cell & 16 & 5.6 \\
\hline Others & 13 & 4.6 \\
\hline \multicolumn{3}{|c|}{ Pathological lymph node status } \\
\hline No & 230 & 81.0 \\
\hline N1 & 22 & 7.7 \\
\hline $\mathrm{N} 2$ & 32 & 11.3 \\
\hline \multicolumn{3}{|l|}{ Pathological stage } \\
\hline IA & 120 & 42.2 \\
\hline IB & 82 & 28.9 \\
\hline $\mathrm{II}(\mathrm{A}+\mathrm{B})$ & 42 & 14.8 \\
\hline $\mathrm{III}(\mathrm{A}+\mathrm{B})$ & 40 & 14.1 \\
\hline \multicolumn{3}{|l|}{ Surgical procedure } \\
\hline Pneumonectomy & 2 & 0.7 \\
\hline Lobectomy & 211 & 74.3 \\
\hline Segmentectomy & 30 & 10.6 \\
\hline Wedge resection & 41 & 14.4 \\
\hline
\end{tabular}

in 230 patients $(81.0 \%)$, and $\mathrm{N} 1$ or $\mathrm{N} 2$ disease in 54 patients $(19.0 \%)$. The median follow-up period was 33.9 months (range 5-72 months).

\section{Pathological characteristics in relation to the SUV- E, SUV-D, and the RI}

Table 2 shows the SUV-E, SUV-D and RI values in relation to the pathological findings. The mean SUV-E was 7.3 (range 0-30.7) and the mean SUV-D was 8.9 (range 0-35.7). In relation to the histological type, the SUV-E and -D values were significantly higher in the non-adenocarcinoma group than in the adenocarcinoma group. The SUV-E and -D values were significantly higher in cases with a large tumor sizes $(>31 \mathrm{~mm})$, moderate or poor tumor differentiation grade, and pathological lymph node metastasis, lymphatic invasion, or vascular invasion, than in those with small tumor sizes $(<30 \mathrm{~mm})$, well-differentiated tumors, and no pathological lymph node metastasis, lymphatic invasion, or vascular invasion. The mean RI was $21.1 \%$ (range -23.1 to $214.0 \%$ ). The RI values were significantly higher in the cases with non-adenocarcinomas, moderate or poor tumor differentiation grade, and pathological lymph node metastasis, or vascular invasion than in those with adenocarcinoma, well-differentiated tumors, and no pathological lymph node metastasis or vascular invasion.

\section{Clinicopathological characteristics in relation to the RI}

We categorized the 284 patients according to the RI: $\mathrm{RI} \leq 0$ (Group A; $n=49: 17.3 \%)$ or RI $>0$ (Group B; $n=235)$. Group A had lower SUV-E $(p<0.001)$ and SUV-D $(p<0.001)$ values, a smaller tumor size ( $p=0.001)$, well differentiation tumor grade $(p=0.001)$, and a smaller percentages of patients with lymph node metastasis $(p=0.001)$, pleural invasion $(p<0.001)$, lymphatic invasion $(p<0.001)$, and vascular invasion $(p<0.001)$ than Group $\mathrm{B}$, while no significant association was observed with the age $(p=0.381)$, or sex $(p=0.339)$ (Table 3). It was particularly noteworthy that lymph node metastasis was not confirmed histopathologically in any of the patients with $\mathrm{RI} \leq 0$. A scattergram showed the SUV-E and SUV-D values in relation to the presence/absence of lymph node metastasis (Fig. 1). A significant positive correlation was observed between the SUV-E and SUV-D ( $r=0.971, P<0.001$ ), and none of the all cases with $\mathrm{RI} \leq 0$ had lymph node metastasis irrespective of the SUV-E or SUV-D value.

\section{Histological Characteristics of the cases showing RI $\leq 0$ on DTP FDG-PET}

Table 4 shows the SUV-E, SUV-D, and RI values in relation to the histological subtype of NSCLC in patients with $\mathrm{RI} \leq 0$. The most frequent subtype was lepidic-predominant invasive adenocarcinoma $(n=24)$, followed in frequency by papillary-predominant invasive adenocarcinoma $(n=8)$, acinar-predominant invasive adenocarcinoma $(n=6)$, invasive mucinous adenocarcinoma $(n=6)$, and squamous cell carcinoma $(n=5)$. The SUVE and SUV-D values of acinar-predominant invasive adenocarcinoma and squamous cell carcinoma were higher than those of lepidic-predominant invasive adenocarcinoma. The RI of squamous cell carcinoma was higher than that of lepidic- predominant invasive adenocarcinoma.

\section{Analysis of the prognosis}

Univariate analysis identified the age, tumor size, nodal metastasis, tumor differentiation grade, SUV-E, SUV-D, and RI as predictors of the RFS. On the other hand, multivariate analysis using a Cox proportional hazard model identified only age $(P<0.001)$ and nodal metastasis $(P<0.001)$, and the RI $(P=0.023)$ as independent predictors of the RFS (Table 5). 
Table 2 Pathological characteristics in relation to the SUV-E, SUV-D, and the RI

\begin{tabular}{|c|c|c|c|}
\hline Characteristics & SUV-E & SUV-D & Retention index $(\%)$ \\
\hline All cases & $7.3 \pm 5.8$ & $8.9 \pm 7.0$ & $21.1 \pm 21.6$ \\
\hline \multicolumn{4}{|l|}{ Histology } \\
\hline Adenocarcinoma & $5.4 \pm 5.0$ & $6.5 \pm 6.1$ & $18.4 \pm 18.8$ \\
\hline Non-adenocarcinoma & $10.9 \pm 5.4$ & $13.4 \pm 6.3$ & $26.0 \pm 25.4$ \\
\hline$p$ value & $<0.001$ & $<0.001$ & 0.010 \\
\hline \multicolumn{4}{|l|}{ Tumor differentiation } \\
\hline Well & $3.3 \pm 3.3$ & $4.0 \pm 4.1$ & $15.3 \pm 19.0$ \\
\hline Moderate/poor & $9.2 \pm 5.7$ & $11.3 \pm 6.9$ & $23.9 \pm 22.2$ \\
\hline$p$ value & $<0.001$ & $<0.001$ & 0.001 \\
\hline \multicolumn{4}{|l|}{ Tumor size } \\
\hline$<30 \mathrm{~mm}$ & $5.0 \pm 4.1$ & $6.2 \pm 5.2$ & $22.0 \pm 25.4$ \\
\hline$>31 \mathrm{~mm}$ & $10.8 \pm 6.2$ & $12.9 \pm 7.4$ & $19.7 \pm 14.2$ \\
\hline$p$ value & $<0.001$ & $<0.001$ & 0.337 \\
\hline \multicolumn{4}{|l|}{ Pathological nodal status } \\
\hline Negative & $6.6 \pm 5.6$ & $8.0 \pm 6.9$ & $19.7 \pm 19.1$ \\
\hline Positive & $10.5 \pm 5.3$ & $13.0 \pm 6.2$ & $27.1 \pm 29.4$ \\
\hline$p$ value & $<0.001$ & $<0.001$ & 0.024 \\
\hline \multicolumn{4}{|l|}{ Lymphatic invasion } \\
\hline Negative & $6.6 \pm 6.0$ & $8.0 \pm 7.2$ & $20.0 \pm 23.2$ \\
\hline Positive & $9.6 \pm 4.6$ & $11.8 \pm 5.5$ & $24.5 \pm 15.1$ \\
\hline$p$ value & $<0.001$ & $<0.001$ & 0.065 \\
\hline \multicolumn{4}{|l|}{ Vascular invasion } \\
\hline Negative & $4.8 \pm 4.8$ & $5.9 \pm 6.0$ & $18.9 \pm 25.5$ \\
\hline Positive & $10.4 \pm 5.4$ & $12.7 \pm 6.3$ & $23.8 \pm 15.1$ \\
\hline$p$ value & $<0.001$ & $<0.001$ & 0.045 \\
\hline
\end{tabular}

\section{Discussion}

This study demonstrated that DTP FDG-PET imaging of the primary tumors can be useful to predict the RFS in NSCLC patients. In addition, this method may also be useful to predict the presence of intrathoracic lymph node metastasis. It was particularly noteworthy that lymph node metastasis was not confirmed histopathologically in any of the patients with RI $\leq 0$ on DTP FDG-PET. In addition, we demonstrated that all the cases with SUV-E value of zero also had SUV-D value of zero. Therefore, delayed scan can be omitted when the early scan does not show FDG accumulation in the tumor. This is the first report of investigation of the significance of decreased FDG uptake in the pulmonary lesions of NSCLC.

The SUV measured on FDG-PET is a semi-quantitative measure of the degree of glucose uptake in a lesion. Determination of the SUVmax of primary lung nodules has been reported to be helpful for distinguishing between malignant and benign tumors, based on the relatively higher values in malignant tumors [17]. In 2001, Kubota et al. reported that most malignant lesions, including primary lung cancers, mediastinal node metastases, and lymphomas, showed higher FDG uptake at $2 \mathrm{~h}$ than at $1 \mathrm{~h}$ after injection of FDG, while normal tissues and benign lesions, except for sarcoidosis, showed a lower uptake at $2 \mathrm{~h}$ than at $1 \mathrm{~h}$ after injection; they proposed that, therefore, FDG-PET scans obtained in the delayed-phase show a better sensitivity than the early-phase images [18]. The SUVmax has been established as a powerful predictor of the treatment outcome in NSCLC patients. However, many factors, such as the patient preparation method, procedure, scan acquisition parameters, image reconstruction method, and data analysis method employed, are known to affect the SUVmax [19, 20]. For example, Westerterp et al. described that the reported variations of up to $30 \%$ in SUV values from 3 institutions could pose a serious problem when performing multicenter studies [21]. In fact, differing values of the SUVmax for detecting lymph node metastasis have been reported from different studies. To overcome this disadvantage, we used the RI of the SUVmax using DTP FDG-PET. RI is a relative scale, and therefore does not require standardization. Based on these findings, we investigated the impact of RI on the prognosis in NSCLC patients. 
Table 3 Clinico-pathological characteristics in relation to the RI

\begin{tabular}{|c|c|c|c|c|}
\hline Characteristics & $N$ & $\mathrm{RI} \leq 0$ & $\mathrm{RI}>0$ & $P$ value \\
\hline Patients (number) & 284 & 49 & 235 & \\
\hline SUV-E (mean) & & 2.2 & 8.4 & $<0.001$ \\
\hline SUV-D (mean) & & 2.0 & 10.4 & $<0.001$ \\
\hline Age (mean), year & & 69.5 & 70.6 & 0.381 \\
\hline \multicolumn{5}{|l|}{ Sex } \\
\hline Male & 195 & 31 & 164 & 0.399 \\
\hline Female & 89 & 18 & 71 & \\
\hline Histology & & & & $<0.001$ \\
\hline Adenocarcinoma & 184 & 44 & 140 & \\
\hline Non-adenocarcinoma & 100 & 5 & 95 & \\
\hline Tumor size (mean), mm & & 22.5 & 30.5 & 0.001 \\
\hline Pathological nodal status & & & & $<0.001$ \\
\hline Negative & 230 & 49 & 181 & \\
\hline Positive & 54 & 0 & 54 & \\
\hline Tumor differentiation & & & & $<0.001$ \\
\hline Well & 92 & 34 & 58 & \\
\hline Moderate & 110 & 12 & 98 & \\
\hline Poor & 82 & 3 & 79 & \\
\hline Pleural invasion & & & & $<0.001$ \\
\hline Negative & 185 & 44 & 141 & \\
\hline Positive & 99 & 5 & 94 & \\
\hline Lymphatic invasion & & & & $<0.001$ \\
\hline Negative & 214 & 47 & 167 & \\
\hline Positive & 70 & 2 & 68 & \\
\hline \multicolumn{5}{|l|}{ Vascular invasion } \\
\hline Negative & 157 & 44 & 113 & $<0.001$ \\
\hline Positive & 127 & 5 & 122 & \\
\hline
\end{tabular}

Three steps are involved in the 18-F FDG accumulation in cancer cells: (1) facilitated diffusion through glucose transport proteins; (2) subsequent phosphorylation by hexokinase isoforms producing FDG-6-phosphate; (3) decreased dephosphorylation [22]. Thus, 18-F FDG accumulation depends basically on the rate of transport through the cell membrane and the activity of hexokinase. Theoretically, the ratio of hexokinase to FDG-6-phosphatase more specifically increases in tumors than in inflammatory lesions, resulting in a gradual accumulation of FDG and a further increase of the SUV on delayed imaging. Therefore, RI may be a better indicator of the malignant potential, whereas the SUV-E represents malignant potential of a tumor [23]. Thus, being a possibly better indicator of malignant potential, RI might be a predictor of the risk of nodal metastasis.

Evaluation of lymph node metastases is important for the staging of lung cancer and for the selection of the most appropriate therapy. Since FDG-PET has been shown to be superior to CT for evaluation of the lymph node status in

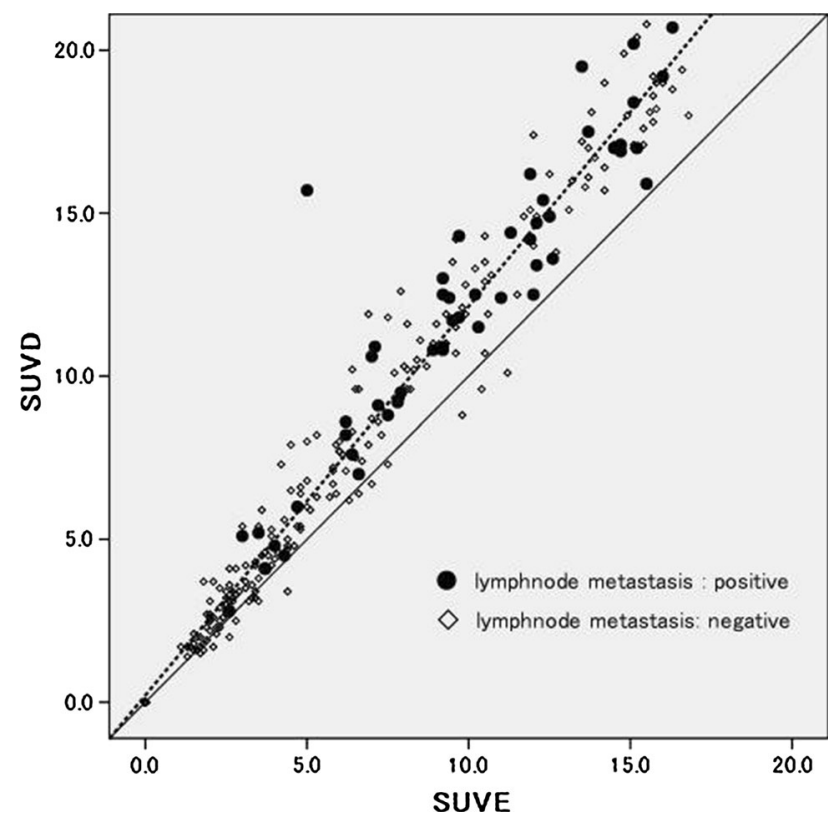

Fig. 1 A scattergram showed that a significant positive correlation was observed between the SUV-E and SUV-D $(r=0.971$, $P<0.001$ ), and none of the all cases with $\mathrm{RI} \leq 0$ had lymph node metastasis

patients with NSCLC, FDG-PET has been increasingly used worldwide [24, 25]. In general, the lymph node status is interpreted qualitatively or quantitatively on FDG-PET scans, based on the tendency towards a higher FDG uptake in metastatic lymph nodes than in non-metastatic lymph nodes $[24,25]$. In addition, recently, several investigators have introduced DTP FDG-PET to improve the accuracy of FDG-PET for nodal staging in cases of NSCLC [11-13]. Based on the results of previous studies, it is known that malignant tumors exhibit increased FDG uptake for several hours, while benign lesions show decreased FDG uptake on DTP FDG-PET [9, 21]. While studies have generally shown favorable accuracy of this modality for nodal staging, this method is rather cumbersome for examining all of the lymph nodes showing FDG accumulation. In this study, we evaluated the primary tumors in NSCLC patients by DTP FDG-PET, which is a simpler and more accurate method than imaging of the number of lymph nodes involved.

To date, DTP FDG-PET has mainly been utilized to improve the accuracy of diagnosis of lung nodules or lymph nodes. Recently, few reports have addressed the possible usefulness of RI in predicting the risk of recurrence or prognosis. Kim et al. reported that RI proved inadequate for predicting the prognosis in terms of the OS and disease-free survival in early stage (stage I and II) NSCLC patients treated by surgical resection [26]. On the other hand, Satoh et al. reported that a higher value of the 
Table 4 Histological characteristics of the cases showing RI $\leq 0$ on DTP FDG$\operatorname{PET}(n=49)$

\begin{tabular}{lrlll}
\hline Characteristics & $n$ & SUV-E & SUV-D & Retention index (\%) \\
\hline Lepidic-predominant invasive AD & 24 & 0.98 & 0.93 & -2.1 \\
Papillary predominant invasive AD & 8 & 2.25 & 2.01 & -5.5 \\
Acinar-predominant invasive AD & 6 & $3.00^{*}$ & $2.85^{*}$ & -3.1 \\
Invasive mucinous AD & 6 & 1.83 & 1.78 & -1.8 \\
Squamous cell carcinoma & 5 & $7.20^{*}$ & $6.58^{*}$ & $-8.7^{*}$ \\
\hline
\end{tabular}

$A D$ adenocarcinoma

* SUV-E: Lepidic vs. Acinar, Lepidic vs. Squamous: $P<0.05$

* SUV-D: Lepidic vs. Acinar, Lepidic vs. Squamous: $P<0.05$

* RI: Lepidic vs. Squamous: $P<0.05$

\begin{tabular}{|c|c|c|c|c|c|c|}
\hline & \multicolumn{3}{|c|}{ Univariate } & \multicolumn{3}{|c|}{ Multivariate } \\
\hline & $\mathrm{HR}$ & $95 \% \mathrm{CI}$ & $p$ value & $\mathrm{HR}$ & $95 \% \mathrm{CI}$ & $p$ value \\
\hline \multicolumn{7}{|l|}{ Sex } \\
\hline Male/female & 1.36 & $0.84-2.19$ & 0.209 & & & \\
\hline \multicolumn{7}{|l|}{ Age } \\
\hline$\geq 70 /<70$ & 2.43 & $1.54-3.85$ & $<0.001$ & 2.45 & $1.54-3.92$ & $<0.001$ \\
\hline \multicolumn{7}{|l|}{ Histology } \\
\hline $\mathrm{AD} /$ non $\mathrm{AD}$ & 0.66 & $0.43-1.02$ & 0.064 & & & \\
\hline \multicolumn{7}{|l|}{ Pathological $T$} \\
\hline$\geq 30 \mathrm{~mm} /<30 \mathrm{~mm}$ & 2.11 & $1.37-3.24$ & 0.001 & 1.27 & $0.77-2.01$ & 0.345 \\
\hline \multicolumn{7}{|l|}{ Pathological $N$ factor } \\
\hline Positive/negative & 5.78 & $3.75-8.91$ & $<0.001$ & 4.63 & $2.92-7.36$ & $<0.001$ \\
\hline \multicolumn{7}{|l|}{ Tumor differentiation } \\
\hline Mod + por/well & 2.64 & $1.53-4.55$ & 0.001 & 1.49 & $0.82-2.72$ & 0.194 \\
\hline SUV-E & 1.08 & $1.04-1.11$ & $<0.001$ & 1.20 & $0.96-1.51$ & 0.106 \\
\hline SUV-D & 1.06 & $1.03-1.09$ & $<0.001$ & 0.87 & $0.72-1.04$ & 0.117 \\
\hline \multicolumn{7}{|l|}{ RI-SUVmax } \\
\hline $\mathrm{RI}>0 / \mathrm{RI} \leq 0$ & 6.93 & $2.19-21.95$ & 0.001 & 4.03 & $1.19-13.63$ & 0.025 \\
\hline
\end{tabular}

Table 5 Analysis of the prognosis this method may also be useful to predict the presence/ absence of intrathoracic lymph node metastasis.

\section{Compliance with ethical standards}

Conflict of interest The authors have no competing interests to declare.

Open Access This article is distributed under the terms of the Creative Commons Attribution 4.0 International License (http://crea tivecommons.org/licenses/by/4.0/), which permits unrestricted use, distribution, and reproduction in any medium, provided you give appropriate credit to the original author(s) and the source, provide a link to the Creative Commons license, and indicate if changes were made.

\section{References}

1. Ung YC, Maziak DE, Vanderveen JA, Smith CA, Gulenchyn K,

Lacchetti C, et al. ${ }^{18}$ Fluorodeoxyglucose positron emission
In conclusion, this study demonstrated that DTP FDG-PET imaging of the primary tumors in patients with NSCLC can be useful to predict the RFS in these patients. In addition, 
tomography in the diagnosis and staging of lung cancer: a systematic review. J Natl Cancer Inst. 2007;99:1753-67.

2. Woodard HQ, Bigler RE, Freed B. Expression of tissue isotope distribution. J Nucl Med. 1975;16:958-9.

3. Vansteenkiste JF, Stroobants SG, Dupont PJ, De Leyn PR, Verbeken EK, Deneffe GJ, et al. Prognostic importance of the standardized uptake value on ${ }^{18} \mathrm{~F}$-fluoro-2-deoxy-glucose-positron emission tomography scan in non-small-cell lung cancer: an analysis of 125 cases. J Clin Oncol. 1999;17:3201-6.

4. Nomori H, Watanabe K, Ohtsuka T, Naruke T, Suemasu K, Kobayashi T, et al. Fluorine 18-tagged fluorodeoxyglucose positron emission tomographic scanning to predict lymph node metastasis, invasiveness, or both, in clinical T1 N0 M0 lung adenocarcinoma. J Thorac Cardiovasc Surg. 2004;128:396-401.

5. Cerfolio RJ, Bryant AS, Ohja B, Bartolucci AA. The maximum standardized uptake values on positron emission tomography of a non-small cell lung cancer predict stage, recurrence, and survival. J Thorac Cardiovasc Surg. 2005;130:151-9.

6. Vesselle H, Freeman JD, Wiens L, Stern J, Nguyen HQ, Hawes $\mathrm{SE}$, et al. Fluorodeoxyglucose uptake of primary non-small cell lung cancer at positron emission tomography: new contrary data on prognostic role. Clin Cancer Res. 2007;13:3255-63.

7. Paesmans M, Berghmans T, Dusart M, Garcia C, Hossein-Foucher C, Lafitte JJ, et al. Primary tumor standardized uptake value measured on fluorodeoxyglucose positron emission tomography is of prognostic value for survival in non-small cell lung cancer. J Thorac Oncol. 2010;5:612-9.

8. Matthies A, Hickeson M, Cuchiara A, Alavi A. Dual time point 18F-FDG PET for the evaluation of pulmonary nodules. J Nucl Med. 2002;43:871-5.

9. Demura Y, Tsuchida T, Ishizaki T, Mizuno S, Totani Y, Ameshima S, et al. 18F-FDG accumulation with PET for differentiation between benign and malignant lesions in the thorax. J Nucl Med. 2003;44:540-8.

10. Cheng G, Alavi A, Werner TJ, Del Bello CV, Akers SR. Serial changes of FDG uptake and diagnosis of suspected lung malignancy: a lesion-based analysis. Clin Nucl Med. 2014;39:147-55.

11. Shinya T, Rai K, Okumura Y, Fujiwara K, Matsuo K, Yonei T, et al. Dual-time-point F-18 FDG PET/CT for evaluation of intrathoracic lymph nodes in patients with non-small cell lung cancer. Clin Nucl Med. 2009;34:216-21.

12. Nishiyama Y, Yamamoto Y, Kimura N, Ishikawa S, Sasakawa Y, Ohkawa M. Dual-time-point FDG-PET for evaluation of lymph node metastasis in patients with non-small-cell lung cancer. Ann Nucl Med. 2008;22:245-50.

13. Uesaka D, Demura Y, Ishizaki T, Ameshima S, Miyamori I, Sasaki M, et al. Evaluation of dual-time-point 18F-FDG PET for staging in patients with lung cancer. J Nucl Med. 2008;49: 1606-12.

14. Macdonald K, Searle J, Lyburn I. The role of dual time point FDG PET imaging in the evaluation of solitary pulmonary nodules with an initial standard uptake value less than 2.5 . Clin Radiol. 2011;66:244-50.

15. Travis WD, Brambilla E, Noguchi M, Nicholson AG, Geisinger $\mathrm{KR}$, Yatabe Y, et al. International association for the study of lung cancer/american thoracic society/european respiratory society international multidisciplinary classification of lung adenocarcinoma. J Thorac Oncol. 2011;6:244-85.

16. Sun JS, Park KJ, Sheen SS, Yoon JK, Yoon SN, Lee KB, Hwang SC. Clinical usefulness of the fluorodeoxyglucose (FDG)-PET maximal standardized uptake value (SUV) in combination with CT features for the differentiation of adenocarcinoma with a bronchioloalveolar carcinoma from other subtypes of non-small cell lung cancers. Lung Cancer. 2009;66:205-10.

17. Bryant AS, Cerfolio RJ. The maximum standardized uptake values on integrated FDG-PET/CT is useful in differentiating benign from malignant pulmonary nodules. Ann Thorac Surg. 2006;82:1016-20.

18. Kubota K, Itoh M, Ozaki K, Ono S, Tashiro M, Yamaguchi K, et al. Advantage of delayed whole-body FDG-PET imaging for tumour detection. Eur J Nucl Med. 2001;28:696-703.

19. Boellaard R, Oyen WJ, Hoekstra CJ, Hoekstra OS, Visser EP, Willemsen AT, et al. The Netherlands protocol for standardisation and quantification of FDG whole body PET studies in multicentre trials. Eur J Nucl Med Mol Imaging. 2008;35:2320-33.

20. Boellaard R, Krak NC, Hoekstra OS, Lammertsma AA. Effects of noise, image resolution, and ROI definition on the accuracy of standard uptake values: a simulation study. J Nucl Med. 2004;45: 1519-27.

21. Westerterp M, Pruim J, Oyen W, Hoekstra O, Paans A, Visser E, et al. Quantification of FDG PET studies using standardized uptake values in multicenter trials: effects of image reconstruction, resolution and ROI definition parameters. Eur J Nucl Med Mol Imaging. 2007;34:392-404.

22. Mamede $M$, Higashi $T$, Kitaichi $M$, Ishizu $K$, Ishimori $T$, Nakamoto Y, et al. $\left[{ }^{18}\right.$ F]FDG uptake and PCNA, Glut-1, and Hexokinase-II expressions in cancers and iLesions of the lung. Neoplasia. 2005;7:369-79.

23. Satoh Y, Nambu A, Onishi H, Sawada E, Tominaga L, Kuriyama $\mathrm{K}$, et al. Value of dual time point F-18 FDG-PET/CT imaging for the evaluation of prognosis and risk factors for recurrence in patients with stage I non-small cell lung cancer treated with stereotactic body radiation therapy. Eur J Radiol. 2012;81: 3530-4.

24. Gould MK, Kuschner WG, Rydzak CE, Maclean CC, Demas AN, Shigemitsu $\mathrm{H}$, et al. Test performance of positron emission tomography and computed tomography for mediastinal staging in patients with non-small-cell lung cancer: a meta-analysis. Ann Intern Med. 2003;139:879-92.

25. Scott WJ, Gobar LS, Terry JD, Dewan NA, Sunderland JJ. Mediastinal lymph node staging of non-small-cell lung cancer: a prospective comparison of computed tomography and positron emission tomography. J Thorac Cardiovasc Surg. 1996;111: $642-8$.

26. Kim SJ, Kim YK, Kim IJ, Kim YD, Lee MK. Limited prognostic value of dual time point F-18 FDG PET/CT in patients with early stage (stage I \& II) non-small cell lung cancer (NSCLC). Clin Nucl Med. 2011;36:434-9. 